\title{
Preparing and probing atomic number states with an atom interferometer
}

\author{
J. Sebby-Strabley, B. L. Brown, M. Anderlini.* P. J. Lee, W. D. Phillips, and J. V. Portd \\ Joint Quantum Institute, National Institute of Standards and Technology, \\ and University of Maryland, Gaithersburg, Maryland 20899, USA \\ P. R. Johnson \\ Physics Program, American University, Washington D.C. 20016 and \\ National Institute of Standards and Technology, Gaithersburg, Maryland 20899, USA
}

(Dated: October 19, 2018)

\begin{abstract}
We describe the controlled loading and measurement of number-squeezed states and Poisson states of atoms in individual sites of a double well optical lattice. These states are input to an atom interferometer that is realized by symmetrically splitting individual lattice sites into double wells, allowing atoms in individual sites to evolve independently. The two paths then interfere, creating a matter-wave double-slit diffraction pattern. The time evolution of the double-slit diffraction pattern is used to measure the number statistics of the input state. The flexibility of our double well lattice provides a means to detect the presence of empty lattice sites, an important and so far unmeasured factor in determining the purity of a Mott state.
\end{abstract}

PACS numbers: 03.75.Gg, 03.67.-a, 32.80.Pj

The optical beam splitter, with its two input and two output modes, is one of the simplest examples of a two mode quantum system. At the quantum level this fundamental system has interesting, nonclassical behavior such as the quantum interference between correlated, indistinguishable photons [1]. This system becomes even richer when the particles interact. While photons may effectively interact in nonlinear media, the atom optics ana$\log$ is naturally interacting. The two mode beam splitter has already been applied in atom optics experiments; several experiments have split a trapped Bose-Einstein condensate (BEC) by raising a barrier to separate the condensate into two independent condensates $[2,3,4,4,5]$. In these experiments the number of atoms is large, in a regime where few-particle quantum interference effects cannot be seen. Here we demonstrate a new few-atom quantum "beam splitter" [6] and use it to create and analyze classical and nonclassical states, resulting in interesting few-atom quantum effects. The ability to create and analyze such states provides a probe of many-body states in a lattice, a platform for fundamental studies of fewparticle, interacting systems, and is of paramount importance for quantum computation with neutral atoms.

We realize an atomic analog of the optical two-mode quantum beam splitter with ${ }^{87} \mathrm{Rb}$ atoms loaded into an optical lattice of double wells [7]. This 3D lattice has a unit cell that can be dynamically transformed between single well and double well configurations (Fig. 17). In analogy with the optical beam splitter, the input and output modes in this lattice are either the ground $|g\rangle$ and first excited $|e\rangle$ state of the single well or the ground state of the left $|L\rangle$ and right $|R\rangle$ sites of the double well. Dynamically switching between these two configurations effectively creates an array of "beam splitters" (BS) coupling these modes (in the limit that higher modes are not (a)

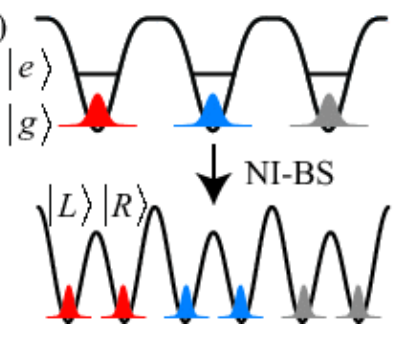

(b)

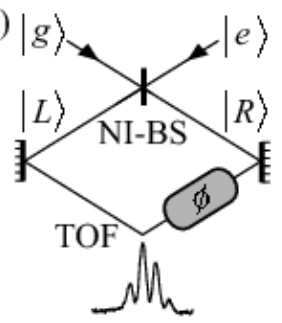

FIG. 1: (color online) (a) Action of a double well beam splitter. Different colors indicate the lack of phase coherence between double well sites. (b) Optical analog of our atom interferometer with an example diffraction pattern resulting from a single input mode.

excited). The interactions between atoms and our control of the beam splitter time scale extends the possible actions beyond those of a traditional beam splitter.

The speed at which the beam splitter is applied to the atoms determines whether interactions play a role in the splitting: if the BS is applied quickly compared to interaction energies, the interactions are unimportant. This "non-interacting" beam splitter (NI-BS) is analogous to the linear optics case: atoms in a single input mode (for example, $|g\rangle$ ) are divided into two output modes (here, $|L\rangle$ and $|R\rangle)$ according to binomial statistics. In contrast if the BS is applied slowly compared to interaction energies, the interactions change the statistics of the distribution between output modes. This "interacting" beam splitter (I-BS) has no simple optical analog. We use the NI-BS and I-BS to create and analyze classical Poisson states and interesting nonclassical few-atom squeezed states. The ability to controllably and reliably load high fidelity Fock states is attractive for applications in quantum information, including the implementation of a two-qubit operation in an optical lattice. 

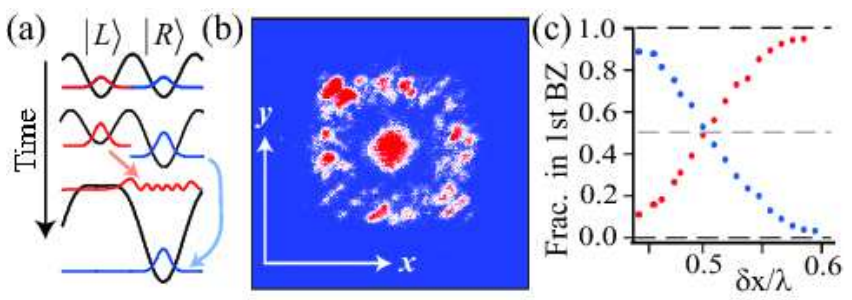

FIG. 2: (color online) (a) Dumping the population initially in $|L\rangle$ into highly excited, possibly unbound bands. (b) The resulting TOF image: the population initially in $|R\rangle$ appears in the first BZ of the $\lambda$-lattice; the population initially in $|L\rangle$ appears in higher BZs as a ring around the first BZ. (c) Calibration of the BS in Fig. 1. After applying the BS at a given value of $\delta x / \lambda$, the population in the $|L\rangle$ (red) or $|R\rangle$ (blue) sites are measured by dumping the other site and measuring the fraction of atoms remaining in the first BZ. The value of $\delta x / \lambda$ where the two curves cross corresponds to a $50 / 50 \mathrm{BS}$.

We probe these states using atom interferometry (Fig. 11). The NI-BS symmetrically and coherently splits a single lattice site into a double well. Atoms in individual sites of a double well evolve independently, analogous to the individual arms of an interferometer. We interfere the two paths by releasing the atoms and allowing them to expand and overlap during time-of-flight (TOF) The interference from a single pair of sites results in a double-slit diffraction pattern. In this experiment the initial states were prepared to have no phase coherence between unit cells; thus the observed image is an incoherent sum of double-slit interference patterns from each of the double wells.

The input states were created and analyzed in a double well optical lattice [7]. We applied this lattice to a BEC of ${ }^{87} \mathrm{Rb}$ atoms in $5 S_{1 / 2}\left|F=1, m_{F}=-1\right\rangle$ created as in [8]. The lattice comprises two independent $2 \mathrm{D}$ lattices having periodicity along $\hat{x}$ of either $\lambda$ or $\lambda / 2$ (Fig. 13) where $\lambda=815 \mathrm{~nm}$ which is red-detuned from the ${ }^{87} \mathrm{Rb} \mathrm{D}$-lines. The relative position $\delta x$ of the " $\lambda$-lattice" and the " $\lambda / 2$-lattice" and their intensities can be dynamically controlled. Transforming from the $\lambda$-lattice to a predominantly $\lambda / 2$-lattice with the minima of the $\lambda / 2$-lattice symmetrically straddling the minima of the $\lambda$-lattice accomplishes the splitting shown in Fig. 1 We use an independent 1D "vertical lattice" in the $\hat{z}$ direction to confine atoms to sites of a 3D lattice. The double well and vertical lattices are focused to $1 / e^{2}$ Gaussian beam radii of $170 \mu \mathrm{m}$ and $250 \mu \mathrm{m}$ respectively and have a relative detuning of $160 \mathrm{MHz}$. The final depths of the $\lambda / 2$-lattice and vertical lattice are $\approx 30 E_{R}$ and $\approx 40 E_{R}$, respectively $\left(E_{R}=\hbar^{2} k^{2} /(2 m)=h \times 3.5 \mathrm{kHz}, k=2 \pi / \lambda\right.$, $m$ is the ${ }^{87} \mathrm{Rb}$ mass). The atomic momentum distribution in the $x, y$-plane is absorption-imaged after TOF.

We measure the populations in the two output ports, $|L\rangle$ and $|R\rangle$, by selectively imparting energy to atoms in one of the two sites, which separates the populations in
TOF (Fig. 2). Transforming to the $\lambda$-lattice (in $300 \mu \mathrm{s}$ ) with its minimum centered over the $|R\rangle$ site, imparts energy to $|L\rangle$ atoms; $|R\rangle$ atoms remain in the ground state of the $\lambda$-lattice (Fig. 2a). The final states' Brillouin zones (BZs), which are filled, are observed (see for instance [7, 9]) by turning off the lattice in $500 \mu$ s and imaging after TOF (Fig. 2 b). The atoms in the center of the image correspond to the filled first BZ of the $\lambda$-lattice 7] and were originally in $|R\rangle$. The atoms in the ring correspond to higher BZs and were originally in $|L\rangle$. This method allows for direct measurement of the atom population in $|L\rangle$ and $|R\rangle$. Using this method we can accurately calibrate the relative position of the two lattices that gives equal splitting of the NI-BS (shown in Fig. 2r). This 50/50 BS was used for all experiments described here.

To prepare number squeezed states with $N=1$ atom in $|g\rangle$ of the $\lambda$-lattice sites, we used a two-stage "slow" loading procedure. We started with $\approx 2 \times 10^{4}$ atoms from a BEC with no discernable uncondensed fraction in a magnetic trap with $\omega_{\perp} / 2 \pi=24 \mathrm{~Hz}$ and $\omega_{\|} / 2 \pi=8 \mathrm{~Hz}$ and Thomas Fermi (TF) radii of $\approx 13 \mu \mathrm{m}$ and $\approx 32 \mu \mathrm{m}$, respectively. The vertical lattice was slowly raised in $250 \mathrm{~ms}$, forming an array of 2D "pancakes." After the first $100 \mathrm{~ms}$ of this loading, we raised the $\lambda$-lattice in the remaining $150 \mathrm{~ms}$ [15]. Ideally, at zero temperature, sufficiently slow loading produces a vertical array of $2 \mathrm{D}$ Mott insulating systems with one atom in $|g\rangle$ of each $\lambda$-site. To prepare Poisson states with $\langle N\rangle \approx 1$ in $|g\rangle$, we used the same procedure, except we loaded both lattices quickly $(500 \mu \mathrm{s})$. This "fast" load is adiabatic only compared to band excitation [7]. We destroyed the phase coherence between atoms in different $\lambda$-sites by holding atoms in the lattice for $5 \mathrm{~ms}$. After preparing either of these initial states, we applied the NI-BS by transforming to the $\lambda / 2$-lattice in $300 \mu \mathrm{s}$, a timescale fast enough to maintain coherence within a double well, yet slow enough to avoid vibrational excitation. After a variable hold time $t$, the atoms were released and imaged after $13 \mathrm{~ms}$ TOF.

Fig. 3a shows an example double-slit interference pattern. We extract the fringe visibility $C(t)$ and the spatial phase $\phi(t)$ by fitting the profile to:

$$
F(x, t)=A e^{-\frac{\left(x-x_{0}\right)^{2}}{2 \sigma^{2}}}\left[1+C(t) \cos \left(\frac{x-x_{0}}{\Delta}+\phi(t)\right)\right],
$$

where $\sigma$ is determined by the width of the ground state wavefunction in a $\lambda$-site, and $2 \pi \Delta$ is the fringe spacing. We use $C(t)$ to measure the number statistics of our input states. $\phi(t)$ evolves linearly due to an energy offset (tilt) $V$ between the sites within a double well [16] (see Fig. 3b).

The NI-beam splitter binomially splits $N$ atoms in each $\lambda$-lattice site into superpositions of states $\left|n_{L}, n_{R}\right\rangle$, with $n_{L}$ atoms in $|L\rangle$ and $n_{R}=N-n_{L}$ atoms in $|R\rangle$, resulting in the time dependent wavefunction

$$
|\psi(t)\rangle=\sum_{n_{L}=0}^{N} c\left(n_{L}, n_{R}\right)\left|n_{L}, n_{R}\right\rangle e^{-i \omega\left(n_{L}, n_{R}\right) t}
$$



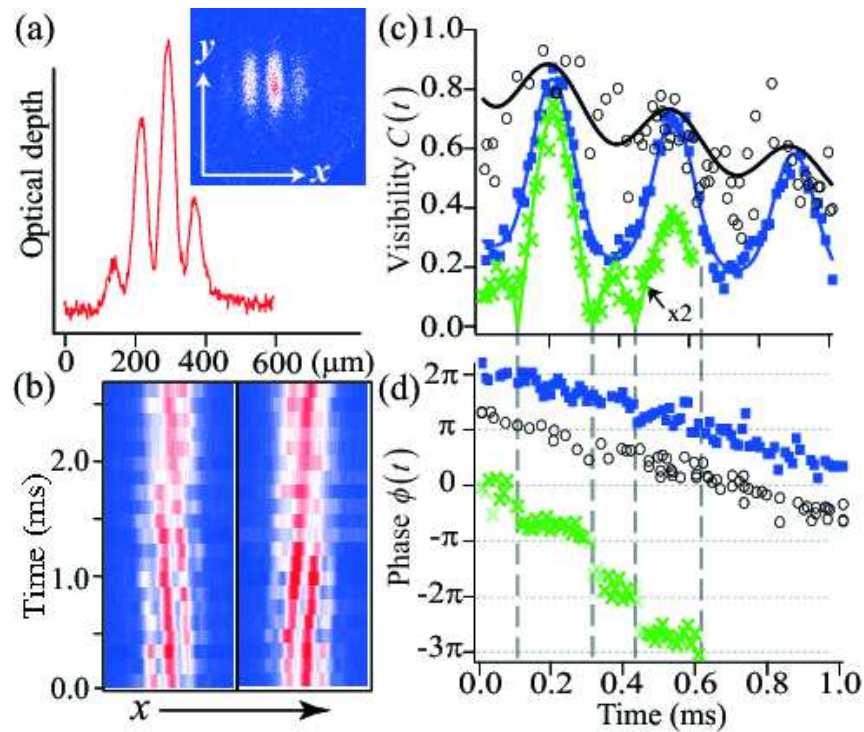

FIG. 3: (color online) (a) A double-slit interference pattern (inset) and integrated density profile after $13 \mathrm{~ms}$ TOF. (b) Example $y$-integrated diffraction pattern as a function of time for tilts $V=4.90(3) \mathrm{kHz}$ (left) and $V=-4.90(3) \mathrm{kHz}$ (right). (c) $C(t)$ for $N=1$ (black $\circ), N=2($ green $\times)$, and $\langle N\rangle=1$ (blue $\mathbf{\square}$ ). Solid lines are a fit to Eq. 2 Note: The plotted $C(t)$ for $N=2$ was scaled by 2 . (d) $\phi(t)$ for the cases shown in (c). The $\phi(t)$ points which were derived from a signal with $2 \times C(t)<0.1$ are shaded in light green. $\phi(t=0)=0$ for all three cases, but each has been shifted for visual clarity.

where $\left|c\left(n_{L}, n_{R}\right)\right|^{2}=\left(\frac{1}{2}\right)^{N}\left(N ! / n_{L} ! n_{R} !\right)$. In the twomode approximation interactions and tilt determine $\hbar \omega\left(n_{L}, n_{R}\right)=n_{L} V+(1 / 2) U n_{L}\left(n_{L}-1\right)+(1 / 2) U n_{R}\left(n_{R}-\right.$ $1)$, where $U$ is the on-site interaction energy per particle. For our system $U / h \approx 3 \mathrm{kHz}$.

In the absence of interactions and inhomogeneities, $|\psi\rangle$ always factors into products of single particle states, $|\psi\rangle=\left(|1,0\rangle+e^{i \omega_{\text {tilt }} t}|0,1\rangle\right)^{N}$, where $\omega_{\text {tilt }}=V / \hbar$. All the terms in Eq. 1 constructively interfere and $C(t)$ is maximized and constant. When interactions are included, $|\psi\rangle$ is not always factorable, and $C(t)$ (for atoms in the ground band) is modulated by $\left|\cos ^{N-1}(U t / \hbar)\right|$ 10, 11]. Summed over a distribution of site occupation numbers, the total $C(t)$ is given by

$$
C(t)=\frac{e^{-\Gamma t}}{\sum_{N=1}^{N_{\max }} f_{N} N}\left|\sum_{N=1}^{N_{\max }} f_{N} N \cos ^{N-1}(U t / \hbar)\right|
$$

where $f_{N}$ is the fraction of $\lambda$-sites with $N$ atoms (for our case we limited $\left.N_{\max }=4\right), \sum f_{N}=1$, and $\Gamma$ is an empirical dephasing rate that we attribute to lattice inhomogeneities. Fitting $C(t)$ with Eq. 2 we can extract the relative fractions $f_{N}$.

For the slow loading case with $N_{\mathrm{BEC}} \leq 2 \times 10^{4}$ (average filling $\leq 1$ per site) we expect to have a Mott state; we measure $C(t)$ to be approximately constant (see Fig. 35), indicating that occupied $\lambda$-lattice sites have $N=1$.
$(C(t)$ shows a slow decay, which we attribute to tilt inhomogeneities.) Extracting $f_{N}$ from $C(t)$, we determine $f_{1}=0.94(6)$. (As shown in Fig. 4a, $f_{1}$ was optimized at a load time of $150 \mathrm{~ms}$.) The uncertainty in $f_{1}$ is dominated by the shot-to-shot scatter in the double-slit visibility $C$, which is significantly larger than for fast loading. We do not understand this increased noise, but note that the shot-to-shot scatter in $\phi$ (Fig. $3 \mathrm{~d} d)$ is not increased. This suggests an increased sensitivity to an uncontrolled initial condition, e.g., number or temperature.

The near-zero value of $f_{2}$ indicates a strongly numbersqueezed initial state, as expected in a Mott insulator (compared to the Poisson value of 0.3 for average filling of unity, ignoring $f_{0}$ ). The value of $f_{1}$ does not, however, necessarily represent the fidelity of the Mott state since this measurement is insensitive to sites with $N=0$. Assuming $f_{1}=0.94$ and $f_{2}=0.06$ and a homogeneous system, we can roughly estimate the temperature $T$ in the $\lambda$-lattice from $f_{2}=e^{-U / k_{B} T}$. This gives $T \leq 0.36 U / k_{B} \simeq 50 \mathrm{nK}$.

For the fast loading case where we expect a Poisson distribution of site occupation numbers, there can be $N>1$ atoms at any $\lambda$-site. In this case we find that $C(t)$ shows distinct collapses and revivals, caused by interactions (see Fig. 3r). Only at integer multiples $l$ of $T_{\text {rev }}=h / U$ does the wavefunction within a site factor: $\psi=\left(|1,0\rangle+e^{i l \omega_{\text {tilt }} T_{\text {rev }}}|0,1\rangle\right)^{N}$. We observe revivals in $C(t)$ with a period of $\approx 350 \mu \mathrm{s}$, in good agreement with our calculated value of $U / h=2.88 \mathrm{kHz}$. While similar to the interaction-induced collapse and revival seen in [12], the interference here is between the sites of a double well and is created by a topology change in the lattice, rather than between sites extending over the entire lattice and created by changing the lattice depth. This allows us to see interference even in the absence of global coherence, as evidenced by the interference pattern generated from the highly squeezed $N=1$ slow load state.

Using Eq. 2 to fit the fast load $C(t)$, we extract the number distribution of atoms in $\lambda$-lattice sites. By fitting that distribution to an average over Poisson distributions whose local mean occupation numbers are proportional to the initial TF-density profile, we extract $\langle N\rangle$, the peak number density of atoms in $\lambda$-lattice sites. This weighted Poissonian distribution fits the data within experimental uncertainty. Fig. 4b shows $\langle N\rangle$ as a function of $N_{\mathrm{BEC}}$ and the power law scaling $\left(N_{\mathrm{BEC}}^{2 / 5}\right)$ for $\langle N\rangle$ expected from the TF-approximation. For large values of $N_{\mathrm{BEC}}$ the data deviates from the trend set by the lower values of $N_{\mathrm{BEC}}$. While not completely understood, this result may be attributed to three-body loss mechanisms and a larger thermal fraction for larger values of $N_{\mathrm{BEC}}$.

We now consider input states with $N=2$ atoms in $|g\rangle$ of the $\lambda$-lattice sites. If we simply loaded the $\lambda$-lattice from our harmonic trap with increased initial peak density, the resulting "shell structure" should produce a sig- 

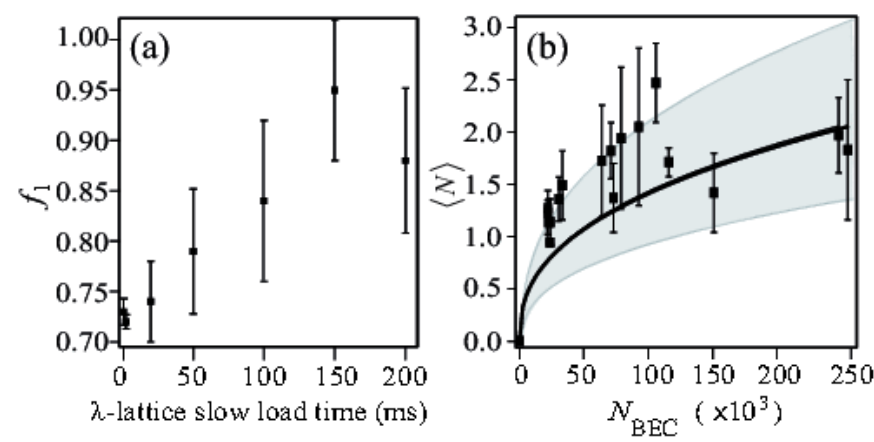

FIG. 4: We use Eq. 2 to fit $C(t)$ for the fast and slow load data. (a) Extracted $f_{1}$ for varied slow load times. The uncertainties are from the fit to Eq. 2] (b) Using Eq. 2 to fit the fast load $C(t)$ we extract values for $f_{1}, f_{2}, f_{3}$ and $f_{4}$. From those coefficients we determine $\langle N\rangle$, the peak number density of atoms in $\lambda$-lattice sites, as a function of $N_{\mathrm{BEC}}$. The line and the grey shaded area are the peak number density scalings predicted by the TF-approximation (with no adjustable parameters) and our estimated uncertainty in the calibration of $N_{\mathrm{BEC}}$ (factor of \pm 1.5 ). The uncertainties on the individual points are from a combination of the uncertainties in $f_{i}$ and from the fit determining $\langle N\rangle$.

nificant number of sites with $N=1$ surrounding the central core of $N=2[13,14]$, limiting the maximum value of $f_{2}$. In principle, the flexibility of the double well lattice can be used to construct a purer $N=2$ state in the $\lambda$ lattice by combining two $\lambda / 2$-lattice sites: we first load the vertical lattice as in the slow load case above, but with an initial number $\left(N_{\mathrm{BEC}} \approx 7 \times 10^{4}\right)$ large enough to produce $\simeq 1$ atom per site in the $\lambda / 2$-lattice. We then raise the $\lambda / 2$-lattice in $100 \mathrm{~ms}$ (ideally, slow enough to make a Mott insulating state with one atom in the ground state of each $\lambda / 2$-site). Finally we adiabatically (in $30 \mathrm{~ms}$ ) combine pairs of $\lambda / 2$-sites into $\lambda$-sites, thus performing an I-BS. The input states are twin Fock states of one atom each in $|L\rangle$ and $|R\rangle$, the two-atom interacting ground state of the $\lambda / 2$-lattice. The output modes are $|g\rangle$ and $|e\rangle$ states of $\lambda$-sites. Interactions and adiabaticity ensure that both atoms go into $|g\rangle$, the two-atom ground state of the $\lambda$-lattice. This "constructed pair" state is then input to our interferometer (split with an NI-BS).

As shown in Fig. 3 c, d we find that after using the constructed pair technique to load the $\lambda$-lattice, $C(t)$ and $\phi(t)$ show unique features which can only be attributed to $\lambda$-sites with $N=2$. As in the fast load case, $C(t)$ collapses and revives, but there are revivals at half the period compared to the fast load case $\left(T_{\text {rev }} / 2\right.$ rather than $\left.T_{\text {rev }}\right)$. Like the slow and fast load cases, $\phi(t)$ evolves linearly as $\omega_{\text {tilt }}$ (here $\approx 0$ ), but here $\phi(t)$ makes clear jumps between revivals. These jumps are $\simeq \pi$, particularly if only the $\phi(t)$ points with $2 \times C(t)>0.1$ are considered. At $l T_{\text {rev }}$ the wavefunction can be factored as $|\psi\rangle=\left(|1,0\rangle+e^{i \omega_{\text {tilt }} T_{\text {rev }}}|0,1\rangle\right)^{2}$, so $C(t)$ revives. Unlike the fast load case, at $l T_{\mathrm{rev}} / 2, C(t)$ also revives but with the wavefunction factored into oppositely-phased single particle states, $|\psi\rangle=\left(|1,0\rangle-e^{i \omega_{\mathrm{tilt}} T_{\mathrm{rev}} / 2}|0,1\rangle\right)^{2}$, giving a $\pi$ phase-shifted interference pattern [10, 11].

Our maximum measured $C$ in Fig. 3 ; for the constructed pairs technique is a factor of $\approx 2$ less than the fast and slow load cases, and our measured $C\left(T_{\text {rev }} / 2\right)$ is lower than $C\left(T_{\text {rev }}\right)$. These differences can be largely explained by the fact that after the I-BS, a large fraction of atoms $(\simeq 30 \%)$ are in $|e\rangle$ (revealed by imaging the $\lambda$-lattice BZ), and make an interference pattern $\pi$ out-of-phase with that made by atoms in $|g\rangle$ [17]. After the NI-BS the incoherent sum of sites with atoms equally in $|g\rangle$ and $|e\rangle$-in total $\simeq 60 \%$ of the atoms - thus contributes $C(t)=0$. $|e\rangle$-population could be caused by I-BS input states such as $\left|n_{L}, n_{R}\right\rangle=|2,0\rangle,|2,1\rangle$, and $|1,0\rangle$, all of which would reflect impurities in the initial $\lambda / 2$-lattice Mott state. The presence of atoms in $|e\rangle$, however, also points to a unique capability of the double well lattice: traditionally it has been impossible to detect empty sites, but by using the constructed pairs technique to load the $\lambda$-lattice and measuring $N=1$, we can detect the presence of empty sites in the initial $\lambda / 2$-lattice.

Using the versatility and control of the double well lattice, future studies can also be focused on creating and exploring more complicated input states with interferometry, such as entangled $|N, 0\rangle+|0, N\rangle$ states. Using the constructed pairs technique but with a NI-BS to combine pairs of $\lambda / 2$-sites to single $\lambda$-sites, we can create $|2,0\rangle+|0,2\rangle$ in the $|g, e\rangle$ basis. This would be the atom optics equivalent of the Hong-Ou-Mandel effect [1].

This work was supported by DTO, ONR, and NASA. The authors thank Jens Kruse, Ian Spielman, and Steve Rolston for contributions to the project. JS-S, BLB, and PJL acknowledge support from the NRC. PRJ acknowledges support from the IC Postdoctoral Program.

* Present address: INFN, Sezione di Firenze, via Sansone 1, I-50019 Sesto Fiorentino (FI), Italy

$\dagger$ Electronic address: trey@nist.gov

[1] C. K. Hong, Z. Y. Ou, L. Mandel , Phys. Rev. Lett. 59, 2044 (1987).

[2] T. Schumm, et al., Nature Physics 1, 57 (2005).

[3] Y. Shin, et al., Phys Rev Lett. 95, 170402 (2005).

[4] Y. Shin, et al., Phys. Rev. Lett. 92, 050405 (2004).

[5] M. Albiez et al., Phys. Rev. Lett. 95, 010402 (2005).

[6] O. Mandel et al., Phys. Rev. Lett. 91, 10407 (2003).

[7] J. Sebby-Strabley et al., Phys. Rev. A 73, 033605 (2006).

[8] S. Peil et al., Phys. Rev. A 67, 051603(R) (2003).

[9] A. Kastberg et al., Phys. Rev. Lett 74, 1542 (1995).

[10] P. R. Johnson, J. Sebby-Strabley, E. Tiesinga, J. V. Porto, and C. J. Williams, in preparation (2007).

[11] D. F. Walls et al., Phil. Trans. R. Soc. A 355, 2393 (1997).

[12] M. Greiner et al., Nature (London) 419, 51 (2002).

[13] S. Folling et al., Phys. Rev. Lett 97, 060403 (2006).

[14] G.K. Campbell et al., Science 313, 649 (2006). 
[15] We load to $\simeq 12 E_{R}$ in $50 \mathrm{~ms}$ then to $\simeq 100 E_{R}$ in $100 \mathrm{~ms}$.

[16] $V$ can result from misalignment of the $\lambda / 2$-lattice [7], which we can in principle compensate with a small amount of the $\lambda$-lattice. The residual $\approx 1 \mathrm{kHz}$ tilt in
Fig. 3d had no effect on the results presented here. [17] Eq. 2 does not account for $|e\rangle$ population. 